Int. J. Morphol.,

36(1):258-261, 2018.

\title{
Pérdida del Dactilograma Durante la Senilidad
}

\author{
Loss of the Dactylogram During Senility
}

Juan Silva'; Mariana Rojas²; Claudia Araya ${ }^{1}$ \& Nicolás Barraza ${ }^{1}$

SILVA, J.; ROJAS, M.; ARAYA, C. \& BARRAZA, N. Pérdida del dactilograma durante la senilidad. Int. J. Morphol., 36(1):258-261, 2018.

RESUMEN: El dactilograma es propio de la piel humana y se observa en la cara volar de dedos, palmas de manos y plantas de pies, caracterizándose por poseer un ordenamiento único y distinguible. Este patrón es fundamental en identificación humana, por ser único, particular, inmutable, perenne, fácil de observar, comparar y almacenar. Se han descrito cambios en la piel senil, pero pocos han observado desvanecimiento de este patrón. Nuestro objetivo fue evidenciar la pérdida del dactilograma en individuos seniles chilenos y determinar si edad y sexo son variables relacionadas con este fenómeno. La muestra abarcó 487 individuos (120 hombres y 367 mujeres) entre 55 y 107 años. En ellos se registró la presencia o ausencia de dactilograma de cara palmar de pulgar. Los datos obtenidos se procesaron estadísticamente. Se determinó que la edad es una variable relacionada con la pérdida del dactilograma y que a mayor edad, mayor probabilidad de que esta característica no esté presente (a los 82 años, el $25 \%$ no presenta dactilograma, a los 88 años aumenta al $50 \%$ y a los 95 años el $75 \%$ ya no presenta dicho patrón). Así mismo, el sexo determinó diferencias importantes en el desvanecimiento del dactilograma, siendo las mujeres más propensas a presentar pérdida de este patrón (51,77\% de la muestra) que los hombres (37,5 \% de la muestra). Pero estos últimos presentan un 42,4\% adicional de riesgo de perder el dactilograma antes que las mujeres. Este trabajo demuestra que el dactilograma se pierde en la senilidad. Hasta ahora este hecho no había sido demostrado. Razón por la cual, este estudio es un aporte significativo en el campo de la morfología y la identificación humana, poniendo de manifiesto que una parte importante de la población chilena ha perdido su identidad basada en las huellas dactilares.

PALABRAS CLAVE: Dermatoglifo; Manifestaciones cutáneas; Envejecimiento de la piel; Medicina forense; Identificación humana.

\section{INTRODUCCIÓN}

El dactilograma es una característica morfológica propia de la piel humana y corresponde al conjunto de crestas, poros sudoríparos y surcos de la superficie cutánea que involucra a epidermis y dermis. Son observables en la cara volar de dedos, palmas de manos y plantas de pies (Fig. 1a) y se caracterizan por poseer un ordenamiento que conforma configuraciones únicas y fácilmente distinguibles (Gibbs, 1967; Silva, 2002; Kücken \& Newell, 2005). Además, son utilizadas ampliamente en la identificación humana desde finales del siglo XIX (Faulds, 1880; Galton, 1892).

Las impresiones producidas por este dactilograma, sobre superficies inertes (botellas, vasos, armas, vidrios, muebles y otros), permiten la formación de patrones pictóricos, conocidos como huellas dactilares (Fig. 1b), y que han sido descritos como: únicos, particulares, inmutables, perennes, fáciles de observar, fáciles de comparar y de almacenar (Galton; Kücken \& Newell, 2004; Teke, 2005).
La dactiloscopia corresponde al estudio científico de estas huellas y tiene por finalidad la identificación infalible de la persona en cuestión (Trujillo, 2007; Guzmán, 2008) y actualmente es el método de identificación más utilizado en el mundo debido a que es un método sencillo, rápido, de bajo costo y muy certero (Beaven, 2003).

Se han descrito una serie de cambios en la piel como parte del proceso de envejecimiento (Bargmann, 1961; Lavker, 1979; Kligman, 1979; Berr et al., 1992; Montagna \& Carlisle, 1979; Lozada Ordoñez \& Rueda, 2010; Silva et al., 2017), pero pocos autores han evidenciado dificultad en la observación de este patrón (Kligman; Berr et al.; Silva et al.).

Observaciones empíricas de profesionales asociados al ámbito forense han puesto en evidencia que algunos individuos seniles chilenos no presentarían dactilograma, siendo dificultoso e inclusive imposible, observar y registrar las figu- 

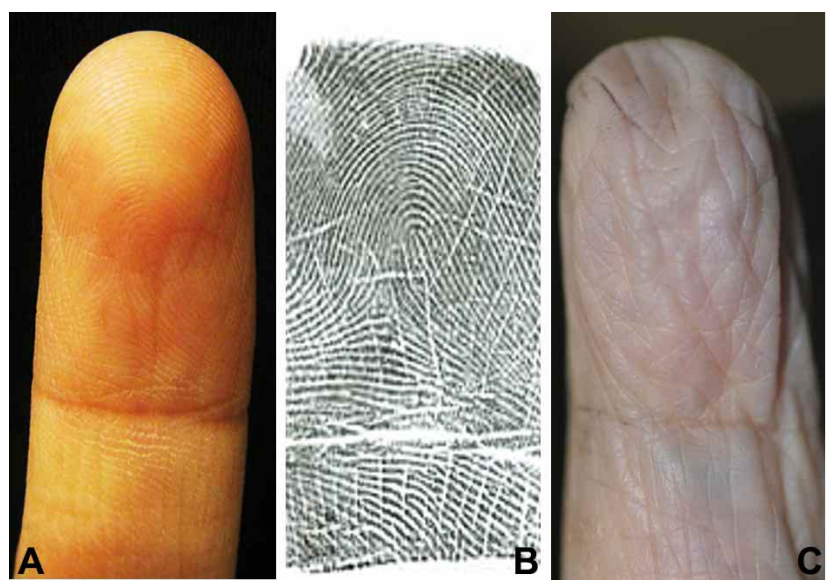

Fig. 1. a) dactilograma presente en la cara volar de dígito. b) huella dactilar producida sobre superficie inerte por dactilograma. c) dígito que evidencia pérdida de dactilograma.

ras características de este patrón morfológico de nuestras manos (Fig. 1c).

El propósito de este trabajo, fue evidenciar la pérdida del dactilograma en individuos seniles chilenos y determinar si la edad y el sexo son variables relacionadas con este fenómeno.

\section{MATERIAL Y MÉTODO}

La muestra abarcó 487 individuos de la ciudad de Santiago de Chile, (120 hombres y 367 mujeres) quienes se encontraban en un rango de edad entre los 55 y los 107 años (Fig. 2). Como criterios de inclusión se consideró que los individuos de la muestra debían presentar sus dedos pulgares y no poseer antecedentes de haber sufrido traumas que pudiesen determinar la pérdida prematura del dactilograma (heridas o quemaduras).

En cada uno de los individuos se observó directamente la presencia o ausencia de dactilograma de la cara palmar del pulgar derecho con lupa entomológica con aumento de 10X e iluminación incorporada.

Los datos obtenidos se recolectaron en formulario ad hoc, y se consideraron las siguientes variables: sexo, edad y presencia o ausencia del dactilograma.

Los datos obtenidos se procesaron con programa STATA 10.0, en el cual se llevó a cabo el análisis estadístico descriptivo. Para la determinación de la edad de desvanecimiento se utilizaron curvas de sobrevida de Kaplan-Meier y para calcular el riesgo asociado al sexo se utilizó regresión de Cox.

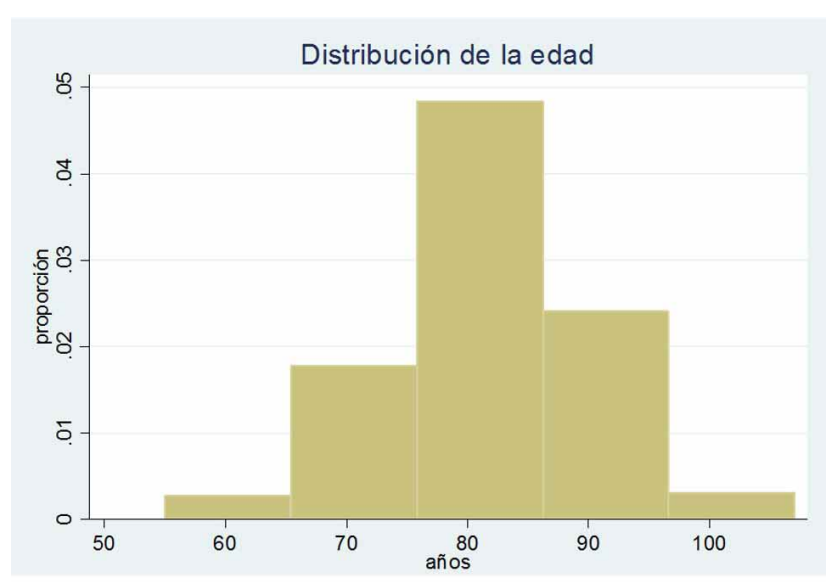

Fig. 2. distribución de la edad de los individuos de la muestra.

Las fotografías se realizaron con cámara digital marca Canon@ modelo PowerShot D30.

\section{RESULTADOS}

De los 487 individuos de la muestra estudiada, 235 individuos no presentaron dactilograma $(48,25 \%$ de la muestra). De los 120 individuos masculinos, 45 no presentaron dactilograma (37,5\% de los hombres) y en el caso de los 367 individuos femeninos, 190 no presentaron dactilograma $(51,77$ $\%$ de las mujeres).

Del estudio realizado sobre esta muestra, sin diferenciar sexo, se observó que a los 82 años, el $25 \%$ de los individuos no presentaron un dactilograma perceptible. A los 88 años el desvanecimiento del dactilograma aumentó al $50 \%$ de la muestra, mientras que a los 95 años el $75 \%$ de los individuos ya no presentan dactilograma.

También fue posible determinar que la edad mínima, a la cuál fue posible observar el desvanecimiento del dactilograma fue de 63 años en el caso de las mujeres y 66 años en el caso de los hombres. En la Figura 3 está representada la curva de sobrevida de Kaplan-Meier, que se obtuvo del total de individuos sin diferenciar sexo.

En la Figura 4, está representada la curva de sobrevida de la identidad diferenciada por sexo (curva de sobrevida de Kaplan-Meier). En esta gráfica se observa, que en el caso de los individuos masculinos, el $25 \%$ de la población ha perdido su dactilograma a los 82 años; el $50 \%$ lo perdió a los 85 años; y el $75 \%$ ya no posee dactilograma a los 94 años. En el caso de las mujeres, el $25 \%$ de la muestra ya no posee dactilograma 
a los 83 años; el $50 \%$ a los 89 años y el $75 \%$ han perdido su identidad a los 95 años.

Del análisis de regresión de Cox es posible concluir que la variable "sexo masculino" está asociada a un $42,4 \%$ adicional de riesgo de perder el dactilograma antes que las mujeres (a menor edad).

Como consecuencia de esto último, es posible sostener que el sexo es una variable relacionada con la probabilidad de perder el dactilograma. Es importante destacar que todos los resultados obtenidos fueron estadísticamente significativos.

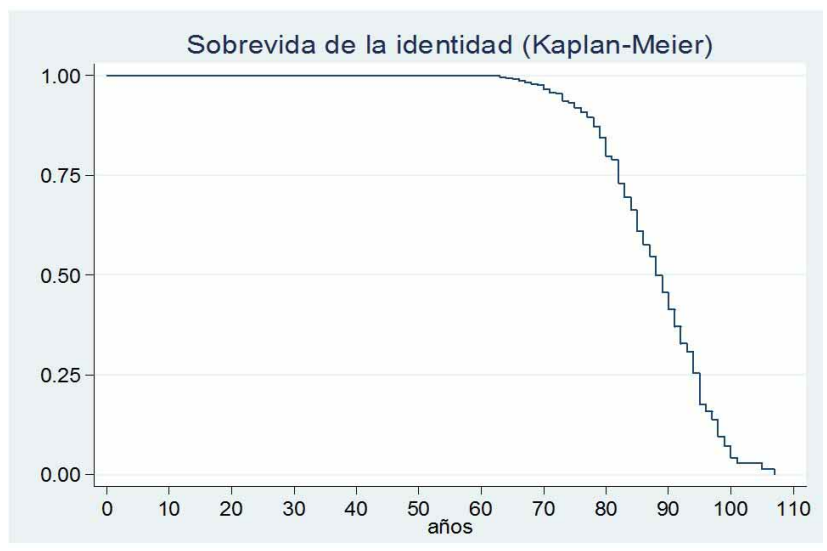

Fig. 3. gráfico que ilustra la curva de sobrevida de la identidad, obtenida en función de la muestra de individuos, sin diferenciar sexo.

\section{DISCUSIÓN}

En este estudio se ha podido constatar que la edad es una variable relacionada con el desvanecimiento y posterior pérdida del dactilograma y que a mayor edad, mayor probabilidad de que esta característica morfológica no esté presente.

Esto es diferente a lo señalado en la literatura relacionada con la dactiloscopia y la criminalística (Beaven; Teke; Trujillo). Pero es concordante con lo descrito por Berr et al. quien afirma que "la observación de esta característica (dactilograma) se hace dificultosa en ancianos".

También pudimos constatar que el sexo de los individuos determinó diferencias en el desvanecimiento de este patrón morfológico, siendo las mujeres más propensas a presentar pérdida de dactilograma $(51,77 \%$ de la muestra) que los hombres (37,5\% de la muestra). Ahora bien, del análisis de la regresión de Cox se pudo concluir que la variable "sexo masculino" está asociada a un 42,4\% adicional de riesgo de perder el dactilograma antes que las mujeres (a menor edad). Esta

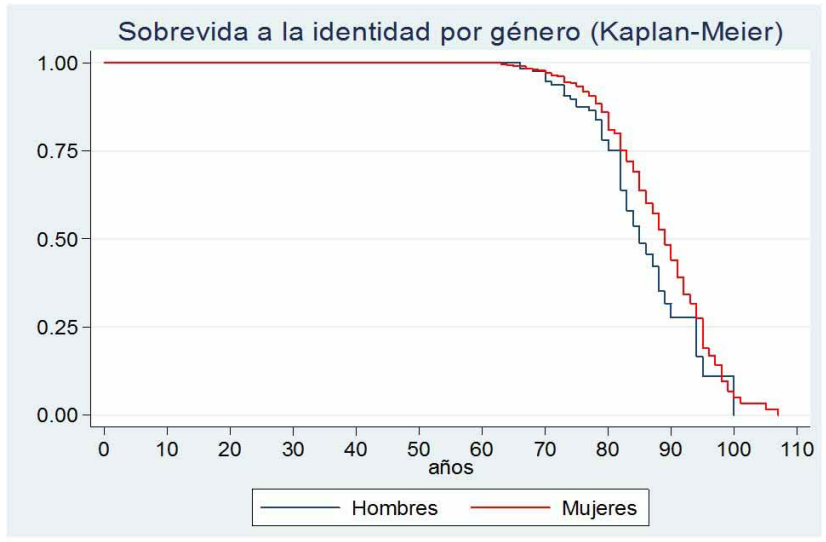

Fig. 4. gráfico que ilustra la curva de sobrevida a la identidad, obtenida en función de la muestra de individuos, diferenciada por sexo.

relación sexo-desvanecimiento, no se encuentra descrita en la literatura.

Finalmente, podemos indicar que este estudio evidencia la pérdida del dactilograma en individuos chilenos de edad avanzada. Hasta ahora este hecho no había sido demostrado científicamente. Debido a lo anterior este estudio es un aporte significativo en el campo de la morfología y la identificación humana, poniendo de manifiesto que una parte importante de la población chilena ha perdido su identidad basada en el método dactiloscópico (por huellas dactilares).

SILVA, J.; ROJAS, M.; ARAYA, C. \& BARRAZA, N. Loss of the dactylogram during senility. Int. J. Morphol., 36(1):248-257, 2018.

SUMMARY: The dactylogram is of one's own human skin and can be observed in the volar face of fingers, palms of hands and soles of feet, it is characterized by having a unique and distinguishable order. This pattern is fundamental in human identification because it is unique, particular, immutable, perennial, easy to observe, compare and store. Changes in senile skin have been described, but few have observed the loss of this pattern. Our objective was to evidence the loss of the dactylogram in Chilean senile individuals and establish if age and sex are variables related to this phenomenon. The sample included 487 individuals (120 men and 367 women) between the ages of 55 to 107 years. In them the presence or absence of dactylogram on the palmar face of the thumbs was recorded. The obtained data were processed statistically. It was determined that age is a variable related to the loss of the dactylogram and that the older the subject, the more likely this feature is not present (At 82 years, $25 \%$ do not present dactylogram, at 88 years it increases to 50 $\%$ and at 95 years, $75 \%$ no longer have such pattern). Likewise, sex determined significant differences in the dactylogram fading, with women being more prone to the loss of this pattern $(51.77 \%$ of the sample) than men (37.5\% of the sample). But the latter have an additional $42.4 \%$ risk of losing the dactylogram earlier than women. 
This work demonstrates that the dactylogram is lost in senility. So far, this fact had not been proven. Therefore, this study is a significant contribution in the field of morphology and human identification, revealing that an important part of the Chilean population has lost its fingerprint-based identity.

KEY WORDS: Dermatoglyph; Cutaneous manifestations; Aging of the skin; Forensic medicine; Human identification.

\section{REFERENCIAS BIBLIOGRÁFICAS}

Bargmann, W. Histología y Anatomía Microscópica Humanas. $3^{\mathrm{a}}$ ed. Barcelona, Editorial Labor, 1961. pp.681-97.

Beaven, C. Huellas Dactilares, los Orígenes de la Dactiloscopia y de la Ciencia de la Identificación Criminal. Barcelona, Editorial Alba, 2003.

Berr, C.; Okra-Podrabinek, N.; Feteanu, D.; Taurand, S.; Hervy, M.; Forette, F.; Piette, F.; Sebag-Lanoe, R. \& Alperovitch, A. Dermatoglyphic patterns in dementia of the Alzheimer type: a case-control study. J. Epidemiol. Community Health, 46(5):512-6, 1992.

Faulds, H. On the skin-Furrows of the hand. Nature, 22:605, 1880.

Galton, F. Finger Prints. Londres, Mc Millan and Co., 1892.

Gibbs, R. C. Fundamentals of dermatoglyphics. Arch. Dermatol., 96(6):7215, 1967.

Guzmán, C. Manual de Criminalística. Buenos Aires, Editorial La Rocca, 2008.

Kligman, A. M. Perspectives and problems in cutaneous gerontology. J. Invest. Dermatol., 73(1):39-46, 1979.

Kücken, M. \& Newell, A. C. A model for fingerprint formation. Europhys. Lett., 68(1):141-6, 2004.

Kücken, M. \& Newell, A. Fingerprint formation. J. Theor. Biol., 235(1):71$83,2005$.

Lavker, R. M. Structural alterations in exposed and unexposed aged skin. $J$. Invest. Dermatol., 73(1):59-66, 1979.

Lozada Ordoñez, S. M. \& Rueda, R. J. Envejecimiento cutáneo. Rev. Asoc. Colomb. Dermatol., 18(1):10-7, 2010.

Montagna, W. \& Carlisle, K. Structural changes in aging human skin. J. Invest. Dermatol., 73(1):47-53, 1979.

Silva, J. El Odontograma Clínico. Un Método Efectivo para la Identificación Médico Legal. Trabajo de investigación requisito para optar al título de Cirujano-Dentista. Santiago de Chile, Universidad de Chile, 2002.

Silva, J.; Rojas, M.; Araya, C. \& Villagra, F. Histological characteristics of the skin of the volar side of the thumb in Chilean individuals with vanishing dactylogram. Int. J. Morphol., 35(3):1107-13, 2017.

Teke, A. Medicina Legal \& Criminalística. Santiago de Chile, Ediciones Jurídicas de Santiago, 2005. pp.193-7.

Trujillo, S. El Estudio Científico de la Dactiloscopía. $2^{\text {a }}$ ed. Ciudad de México, Editorial Limusa, 2007.
Dirección para correspondencia:

Prof. Dr. Juan Luis Silva R.

Facultad de Medicina

Universidad San Sebastian

Providencia

Santiago

CHILE

Email: juan.silva@uss.cl

Recibido : 22-08-2017

Aceptado: 18-10-2017 